SHORT REPORT

\title{
Lamotrigine reduces migraine aura and migraine attacks in patients with migraine with aura
}

\author{
C Lampl, Z Katsarava, H-C Diener, V Limmroth
}

J Neurol Neurosurg Psychiatry 2005;76:1730-1732. doi: 10.1136/jnnp.2005.063750

This study examined the efficacy of lamotrigine in the prevention of migraine aura. Fifty nine patients suffering from migraine with aura received lamotrigine in a controlled three year prospective open study. Treatment response was defined as a reduction of aura frequency each month by at least $50 \%$. Primary endpoint was reached by three quarters of the patients. Lamotrigine significantly reduced both frequency of migraine aura (mean, 1.5 (SD, 0.6) each month before $v 0.4$ (0.7) after treatment; $p<0.001$ ) and aura duration (mean, 27 (SD, 11) minutes before $v 8$ (14) after treatment; $p<0.001)$. Furthermore, more than three quarters of those patients with a reduction of aura symptoms experienced a significant reduction of frequency of migraine attacks (mean, 2.1 (SD, 1.0) each month before $v 1.2$ (1.1) after treatment; $p<0.001$ ). Lamotrigine was highly effective in reducing migraine aura and migraine attacks. The strong correlation between reduction of aura symptoms and migraine attacks stresses the potential role of aura-like events and possibly cortical spreading depression as a trigger for trigeminal vascular activation, and subsequently the development of migraine headaches.

$\mathrm{T}$ en to fifteen percent of all patients with migraine suffer from migraine with aura. For most of these patients the aura phase encompasses visual or sensory deficits for 30 to 60 minutes, and in a few cases for several hours. Using high field functional magnetic resonance imaging with near continuous recording during visual aura, cortical spreading depression (CSD)-like events in the human occipital cortex could be visualised, ${ }^{1}$ confirming the concept of a transient slowly spreading excitation (depolarisation), followed by long lasting depression as the underlying mechanism of migraine aura. Despite the recent development of abortive migraine drugs such as 5-hydroxytryptamine 1B/D agonists, suitable agents for suppressing the frequency and duration of migraine aura have not been identified. Based on concepts of emphasising CSD-like events in migraine pathogenesis, antiglutamatergic strategies have been suggested as a potential avenue for the treatment of migraine auras. ${ }^{2}$

Lamotrigine is a potent sodium channel inhibitor with anti-glutamatergic actions. ${ }^{3}$ In line with other sodium channel inhibitors, lamotrigine did not prevent migraine attacks. ${ }^{4}$ In two short term pilot studies, however, lamotrigine was shown to decrease aura frequency, duration, and symptoms. ${ }^{56}$ Data from one of these studies ${ }^{5}$ indicated that the successful treatment of migraine aura may also reduce migraine attack frequency in this subgroup of patients. Therefore, the aim of our study was to confirm the effects of lamotrigine on migraine aura after longterm treatment in a larger study population, and to investigate whether a reduction in migraine aura frequency is followed by a reduction of migraine attacks.

\section{METHODS AND PATIENTS}

Our study was designed as a prospective, controlled, open, longterm dose titration study. Enrolment took place between February 1995 and December 2000. Informed consent was given by all patients. The study was approved by the local ethics committee (27/95).

\section{Study design \\ Inclusion and exclusion criteria}

Patients between 19 and 60 years of age were eligible if they had suffered from migraine with aura and migraine aura without headache for at least one year (diagnosis according to IHS criteria). One attack each month was the minimum attack frequency for enrolment, with an intensity described as moderate to severe on a four step scale (none, light, moderate, and severe). Exclusion criteria included patients taking prophylactic headache treatment within the three months before the beginning of the trial; previous lamotrigine treatment of epileptic seizures; cardiac, hepatic, or renal diseases; and pregnancy or the risk of pregnancy.

Treatment and evaluation of drug effects

Aura type, frequency, and duration were determined by headache diaries for at least two months before treatment initiation. All patients had to maintain a headache diary and were obliged to document auras and migraine attacks. Control visits were performed each month with evaluation of the diaries, recording of aura symptoms, frequency and duration, and frequency of migraine attacks.

\section{Dose regimen and titration}

Lamotrigine treatment was started with a dose of $25 \mathrm{mg}$ daily during the first month. If no reduction in migraine aura symptoms was seen, the dosage was increased by $25 \mathrm{mg}$ monthly, not exceeding $300 \mathrm{mg} /$ day. If the primary outcome criteria were reached and maintained for at least two months, the lamotrigine dosage was reduced, but not before six months of treatment. When a positive response was maintained for at least two months, lamotrigine was reduced by $25 \mathrm{mg}$, but not below a basic dose of $50 \mathrm{mg} /$ day. If aura symptoms recurred for two months, dosage was increased by $25 \mathrm{mg}$ monthly again until frequency or duration improved. This dosage was the final maintenance dose for the remainder of the study.

\section{Data analysis}

The predetermined primary outcome measure was defined as percentage of patients with a reduction of migraine aura frequency by at least $50 \%$. Secondary outcome measures were the reduction of aura frequency (mean, attacks each month) and the aura duration (mean, minutes) in addition to reduction of frequency of migraine attacks (mean, each

Abbreviations: CSD, cortical spreading depression; $\mathrm{df}$, degrees of freedom 
Table 1 Migraine and aura characteristics, patient demographics, dosages of lamotrigine, and treatment duration

\begin{tabular}{|c|c|c|c|c|}
\hline Parameter & $\mathrm{N} /(\%)$ & Mean & SD & Range \\
\hline Migraine with aura & \multicolumn{4}{|l|}{$56 /(95)$} \\
\hline Aura without headache & \\
\hline Patients total & \multirow{2}{*}{\multicolumn{4}{|c|}{$59 /(100)$}} \\
\hline Type of aura & & & & \\
\hline Visual & \multicolumn{4}{|l|}{$20 /(34)$} \\
\hline Sensory & \multicolumn{4}{|l|}{$14 /(24)$} \\
\hline Visual and sensory & \multicolumn{4}{|l|}{$14 /(24)$} \\
\hline Unilateral paresis & \multicolumn{4}{|l|}{$5 /(8)$} \\
\hline Bilateral paresis arm/leg & \multicolumn{4}{|l|}{$3 /(5)$} \\
\hline Dysarthria/speech deficits & \multicolumn{4}{|c|}{$3 /(5)$} \\
\hline $\operatorname{Sex}(F / M)$ & \multicolumn{4}{|c|}{$35(59) / 24(41)$} \\
\hline Age (years) & 59 & 32.1 & 8.7 & $18-52$ \\
\hline Duration of disease (years) & 59 & 6.3 & 3.8 & $1-20$ \\
\hline Aura frequency (each month) & 59 & 1.5 & 0.6 & $1-3$ \\
\hline Duration of aura (minutes) & 59 & 26.9 & 10.8 & $10-50$ \\
\hline Migraine attack frequency (months) & 56 & 2.1 & 1.0 & $1-6$ \\
\hline Lamotrigine dosage $(\mathrm{mg})$ & 59 & 166.94 & 93.7 & $50-300$ \\
\hline Treatment time to reach primary endpoint (months) & 59 & 4.8 & 2.3 & $2-14$ \\
\hline
\end{tabular}

month). Non-responders were defined as patients with continuing aura symptoms despite treatment for a minimum of three months on a minimum dosage of $100 \mathrm{mg}$ for at least two months.

\section{Statistics}

A paired $t$ test was used to compare mean frequency of migraine aura, mean duration of aura, and mean frequency of migraine attacks. The level of significance was set at 0.5 . Pearson's correlation was used to assess a possible correlation between the reduction in frequency of aura and migraine attacks. All statistics were performed using SPSS10.0.

\section{RESULTS}

\section{Patient population}

Table 1 provides details of migraine and aura presentation, patient demographics, dosages of lamotrigine, and treatment duration.

\section{Treatment effects}

\section{Effects on aura}

A positive response to treatment was seen in 44 of the 59 patients (table 2). Lamotrigine significantly reduced the frequency of migraine aura (mean, 1.5 (SD, 0.6) each month before $v 0.4$ (0.7) after treatment; degrees of freedom $(\mathrm{df})=58 ; t=10.5 ; \mathrm{p}<0.001)$ and the duration of aura

Table 2 Treatment response to lamotrigine

\begin{tabular}{|c|c|c|}
\hline Parameter & $n / N$ & $\%$ \\
\hline Responder (aura)* & $44 / 59$ & 75 \\
\hline Non-responder (aura) & $15 / 59$ & 25 \\
\hline Total & 59 & 100 \\
\hline Responder (migraine attacks) ${ }^{*} \dagger$ & $36 / 56$ & 64 \\
\hline $\begin{array}{l}\text { Patients with significant reduction of } \\
\text { migraine attacks within the responder } \\
\text { group }(n=44)\end{array}$ & $34 / 44$ & 77 \\
\hline $\begin{array}{l}\text { Patients with significant reduction of } \\
\text { migraine attacks within the Non-Responder } \\
\text { group }(n=15)\end{array}$ & $2 / 15$ & 13 \\
\hline
\end{tabular}

Within the group of responders 19 patients reported a significant reduction ( $>50 \%$ ) of the aura; 15 patients did not experience aura for more than 6 months.

*The correlation between the reduction of migraine aura and migraine frequency was highly significant $(p<0.002$, using Pearson's correlation coefficient, 2 sided); treferring only to patients suffering from migraine with aura $(n=56)$. symptoms (mean, 26.9 (SD, 10.8) minutes before $v 8.3$ (13.6) after treatment; $\mathrm{df}=58 ; t=10.8 ; \mathrm{p}<0.001)$. Treatment effects were not different for different types of aura.

\section{Effects on migraine attacks}

The frequency of migraine attacks was significantly reduced also (mean, 2.1 (SD, 1.0) each month before $v 1.2$ (1.1) after treatment; $\mathrm{df}=55 ; t=7.7 ; \mathrm{p}<0.001)$. A significant correlation was found between the reduction in frequency of aura and migraine headache attacks (table 2).

\section{DISCUSSION}

Lamotrigine was highly effective in reducing the frequency and intensity of migraine aura. Furthermore, in more than three quarters of those patients who experienced improvement, a significant decrease in migraine frequency was also seen, whereas this effect was not seen among nonresponders. These data confirm the results of pilot studies conducted with lamotrigine for the treatment of migraine aura, ${ }^{56}$ and suggest that the drug may also be useful in the treatment of patients with more severe types of aura (such as familiar hemiplegic migraine). For most patients, dosages in the range of 75 and $150 \mathrm{mg}$ were sufficient to achieve a consistent response over the entire treatment period. Interestingly, once patients reached their "final" daily dosage, the treatment effect was sustained in almost all patients for the rest of the observation period. The fact that it took almost five months to reach the primary endpoint indicates that patients should be treated for at least six months before this treatment can be considered as ineffective. It also suggests that clinical trials for the prevention of migraine should ideally cover a treatment and evaluation phase of at least six months.

\section{Limitations of our study}

Our study had some limitations. First, there was no placebo group comparison. However, we considered placebo treatment for six or more months not appropriate and unethical. ${ }^{7}$ Second, we did not measure serum concentrations of lamotrigine. Although in several pain studies, a significant association was found between analgesia and plasma lamotrigine concentrations, the dosage in this titration design was guided by the clinical response (reduction of aura symptoms) only. Therefore, measurements were without additional value for the dose finding procedures. 
Lessons for migraine pathophysiology and treatment Recently, Bolay et al demonstrated elegantly that meningeal afferents can be activated by CSD in animals. ${ }^{8}$ The fact that the patients in our study not only experienced a reduction in migraine aura, but also a significant reduction in migraine frequency, suggests that the inhibition of CSD-like events may subsequently prevent migraine headache in this subgroup of patients. Our results also suggest that aura as a possible clinical entity initiates a complex neuronal pathway, resulting in typical migraine headache. In patients with migraine without aura, however, other (still unknown) mechanisms, such as a temporary impairment of the periaqueductual grey, probably play a crucial role in the activation of the trigeminal vascular system. ${ }^{9}$

Therefore, our study may provide the first clinical evidence consistent with the concept that CSD-like events activate the trigeminal vascular system under specific circumstances in the human cortex. Because there is now compelling evidence that CSD-like events may either be caused by extensive glutamate release or reduced clearance from the synaptic cleft, ${ }^{10}$ treatment by downregulating NMDA receptor activity provides an important approach to managing patients with migraine with aura.

\section{Authors' affiliations}

C Lampl, Department of Neurology and Psychiatry, Pain and Headache Centre, Linz General Hospital, 4020 Linz, Austria

Z Katsarava, H-C Diener, V Limmroth, Department of Neurology, Essen University Hospital, Essen, Germany
Competing interests: none declared

Correspondence to: $\operatorname{Dr} C$ Lampl, Department of Neurology and Psychiatry, Pain and Headache Centre, Linz General Hospital, 4020 Linz, Austria; christian.lampl@akh.linz.at

Received 18 January 2005

Revised version received 15 March 2005

Accepted 18 March 2005

\section{REFERENCES}

1 Hadjikhani N, Sanchez Del Rio M, Wu O, et al. Mechanisms of migraine aura revealed by functional MRI in human visual cortex. Proc Natl Acad Sci U S A 2001;98:4687-92.

2 Kaube H, Herzog J, Kaufer T, et al. Aura in some patients with familial hemiplegic migraine can be stopped by intranasal ketamine. Neurology 2000;55:139-41.

3 Wang SJ, Huang CC, Hsu KS, et al. Presynaptic inhibition of excitatory neurotransmission by lamotrigine in the rat amygdalar neurons. Synapse 1996;3:248-55.

4 Steiner TJ, Findley $\sqcup$, Yuen AW. Lamotrigine versus placebo in the prophylaxis of migraine with and without aura. Cephalalgia 1997; 17:109-12.

5 Lampl C, Buzath A, Klinger D, et al. Lamotrigine in the prophylactic treatment of migraine aura-a pilot study. Cephalalgia 1999;19:58-63.

6 D'Andrea G, Granella F, Cadaldini M, et al. Effectiveness of lamotrigine in the prophylaxis of migraine with aura: an open pilot study. Cephalalgia 1999; 19:64-6.

7 Linde M, May A, Limmroth V, et al. Headache masters programme. Ethical aspects of placebo in migraine research. Cephalalgia 2003;23:491-5.

8 Bolay H, Reuter U, Dunn AK, et al. Intrinsic brain activity triggers trigeminal meningeal afferents in a migraine model. Nat Med 2002;8:136-42.

9 Weiller C, May A, Limmroth V, et al. Brain stem activation in spontaneous migraine attacks. Nat Med 1995;1:658-60.

10 Moskowitz MA, Bolay H, Dalkara T. Deciphering migraine mechanisms: clues from familial hemiplegic migraine genotypes. Ann Neurol 2004;55:276-80. 